\title{
Intellectual disability co-occurring with schizophrenia and other psychiatric illness: population-based study
}

\author{
Vera A. Morgan, Helen Leonard, Jenny Bourke and Assen Jablensky
}

\section{Background}

The epidemiology of intellectual disability co-occurring with schizophrenia and other psychiatric illness is poorly understood. The separation of mental health from intellectual disability services has led to a serious underestimation of the prevalence of dual diagnosis, with clinicians ill-equipped to treat affected individuals.

\section{Aims}

To estimate the prevalence of dual diagnosis and describe its clinical profile.

\section{Method}

The Western Australian population-based psychiatric and intellectual disability registers were cross-linked (total $n=245$ 749).

\section{Results}

Overall, $31.7 \%$ of people with an intellectual disability had a psychiatric disorder; $1.8 \%$ of people with a psychiatric illness had an intellectual disability. Schizophrenia, but not bipolar disorder and unipolar depression, was greatly overrepresented among individuals with a dual diagnosis: depending on birth cohort, $3.7-5.2 \%$ of those with intellectual disability had co-occurring schizophrenia. Pervasive developmental disorder was identified through the Intellectual Disability Register and is therefore limited to individuals with intellectual impairment. None the less, pervasive developmental disorder was more common among people with a dual diagnosis than among individuals with intellectual disability alone. Down syndrome was much less prevalent among individuals with a dual diagnosis despite being the most predominant cause of intellectual disability. Individuals with a dual diagnosis had higher mortality rates and were more disabled than those with psychiatric illness alone.

\section{Conclusions}

The facility to combine records across administrative jurisdictions has enhanced our understanding of the epidemiology of dual diagnosis, its clinical manifestations and aetiological implications. In particular, our results are suggestive of a common pathogenesis in intellectual disability co-occurring with schizophrenia.

\section{Declaration of interest}

None. Funding detailed in Acknowledgements.
Intellectual disability (overlapping terms: intellectual handicap, mental retardation, developmental disability and learning disability) covers a variety of clinical presentations, syndromes and underlying pathologies. ${ }^{1}$ Its overall population prevalence is estimated to be approximately $1.0 \%$, but may vary depending on the age structure of the population under investigation owing to differential mortality rates and under-ascertainment in adult populations. ${ }^{2}$ Australian data put the lifetime prevalence for adults aged 15-64 years at $1.3 \%,{ }^{3}$ while in Western Australia it has been estimated at 14 per 1000 live births. ${ }^{4}$ Most studies have found that the risk of psychiatric disorder is increased among people with intellectual disability, although the reported prevalence varies greatly due to methodological and nosological problems which affect the reliability of estimates; these include the criteria used to assign intellectual disability status and level and to ascertain psychiatric morbidity, as well as issues of sample size, source, age and bias. ${ }^{5-7}$ Of underlying significance is the fact that the identification of mental illness in individuals with intellectual disability is difficult. ${ }^{5}$ As a result, the epidemiology of intellectual disability co-occurring with psychiatric illness remains poorly understood. ${ }^{8}$ Some specific associations have been reported. In particular, there appears to be an elevated lifetime risk of schizophrenia among individuals with intellectual disability. In 1938, Penrose reported that $3.8 \%$ of 1280 individuals with intellectual disability had schizophrenia and $1.9 \%$ had affective psychoses; ${ }^{9}$ current estimates still put the risk of schizophrenia in intellectually disabled populations at around $3 \%,{ }^{5,10}$ compared with a lifetime population risk of around $1 \%$.
The current work presents a population-based approach to the study of co-occurring intellectual disability and psychiatric illness - 'dual diagnosis' in its original rather than its more recently acquired meaning of psychiatric illness and comorbid drug and alcohol misuse. ${ }^{3}$ This study has linked two population-based registers maintained in separate administrative health jurisdictions in Western Australia (current population 2.1 million), namely the Mental Health Information System and the Intellectual Disability Register in order to:

(a) estimate the prevalence of psychiatric disorders among people with intellectual disability and, conversely, the prevalence of intellectual disability among people with a psychiatric disorder

(b) describe the disability and service utilisation profile of people with a dual diagnosis.

\section{Method}

The Intellectual Disability Register was established in 1953. As registration is necessary for receipt of services, coverage of the register is good, rising with age. It is estimated to cover over $75 \%$ of Western Australian cases, with under-ascertainment most likely to affect borderline cases. At the time of data linkage, it included 11576 individuals who met eligibility criteria based on American Association on Mental Retardation criteria ${ }^{11}$ including a full-scale IQ score greater than or equal to 2 standard deviations below the population mean in combination with limitations in adaptive behaviours and skills. IQ up to 74 may be included in 
cases of severe limitations in adaptive behaviours and skills. Level of intellectual handicap is recorded as borderline (IQ 70-74), mild (IQ 50-55 to 69), moderate (IQ 35-40 to 50-54), severe (IQ 20-25 to 35-39), profound (IQ $<20-25$ ) or unspecified. The register uses the Heber system to classify the type of intellectual disability. ${ }^{12}$ As this system is quite old, three of the authors (V.M., H.L. and J.B.) rearranged the Heber codes into groups that were more consistent with current classification systems. These groups indicate the likely aetiological basis of the intellectual disability, based broadly on categories developed by Yeargin-Allsopp. ${ }^{13}$ They include: genetic (X-linked, other chromosomal, other), metabolic, teratogenic (congenital infections, chemical agents), central nervous system defect, other birth defect, neonatal/post-neonatal, perinatal and multifactorial causes; associated conditions were also recorded. There is also a field on the register for recording psychiatric comorbidities. The Mental Health Information System is a psychiatric case register that records all in-patient and ambulatory care contacts with public mental health services since 1966, as well as admissions to private hospitals, but not services provided by general practitioners and private psychiatrists; ${ }^{14}$ at the time of linkage it included records for 236973 persons who met the study criteria (outlined below). As the register uses different versions of the ICD classification system ranging from ICD-8 to ICD-10-AM, depending on year of diagnosis, an algorithm was written by the first author to translate all diagnoses into their ICD-9 equivalents. ${ }^{15}$

Individual records on the two registers were linked using probabilistic data matching strategies; the methodology used to link records across Western Australian health registers, and its validation, have been published. ${ }^{16}$ Two diagnostic categories have potential overlap across the two registers. The category pervasive developmental disorder was determined using Heber data on the Intellectual Disability Register only. This conservative approach ensured better uniformity by including only those cases with co-occurring intellectual disability. The vast majority (89\%) of cases of pervasive developmental disorder in the dual-diagnosis group had an ICD-9 diagnosis on the psychiatric register other than childhood psychosis. A person on the Intellectual Disability Register with problem behaviours was not counted as having conduct disorder unless this was recorded on the psychiatric register.

To be selected into the intellectual disability arm of the study, a person had to meet American Association on Mental Retardation criteria. For this study, this was extended to include borderline level of intellectual disability with IQ scores of $70-74$; some $12 \%$ ( $n=1607)$ of all individuals with an intellectual disability were classified as borderline. Individuals on the psychiatric case register with an ICD diagnosis of mental retardation were also classified as intellectually disabled. The criterion for selection into the psychiatric arm of the study was having at least one ICD-9 Chapter 5 (mental disorder) diagnosis. People on the psychiatric register with a diagnosis of mental retardation only were retained as intellectually disabled but not included as having a co-occurring psychiatric illness. If a person had psychiatric comorbidity recorded on the Intellectual Disability Register but no ICD-9 diagnosis on the psychiatric case register they were coded as having a psychiatric illness not otherwise specified. As an individual on the psychiatric case register could have multiple psychiatric diagnoses assigned over time, we selected the last diagnosis recorded on the register as the project diagnosis for that person. The main diagnostic categories used in analysis were: schizophrenia (ICD-9 295), bipolar disorder (ICD-9 296.0 and 296.2-5), and unipolar (major) depression (ICD-9 296.1, 296.6, 296.8 and 296.9), as well as an aggregated category, any non-organic psychosis that combines ICD-9 295-298 including major depression. Dual diagnosis cases were those with both intellectual disability and a psychiatric illness as defined above.

For the purposes of this study, individuals were identified as having a pervasive developmental disorder (autism, Asperger syndrome, Rett syndrome or unspecified) on the basis of such diagnoses being recorded on the Intellectual Disability Register alone. Thus, all identified cases of pervasive developmental disorder had IQs within the intellectual disability range. These individuals were only counted as having a dual diagnosis if they also had an ICD-9 diagnosis of mental illness. Although there was some potential overlap with the ICD-9 code 299 (childhood psychosis) on the psychiatric register, the vast majority (89\%) of individuals with pervasive developmental disorder on the Intellectual Disability Register had a diagnosis of mental illness other than childhood psychosis. Likewise, individuals were identified as having problem behaviours only if they had problem behaviours recorded on the Intellectual Disability Register. Thus, all cases of problem behaviour also had IQs within the intellectual disability range. These individuals were only counted as having a dual diagnosis if they also had an ICD-9 diagnosis of mental illness.

Because cross-linkage of registers identified both duplication of individuals across registers as well as additional cases of intellectual disability, psychiatric disorder or dual diagnosis, the final research database of 245749 persons is not simply the sum of the combined categories on the individual registers.

\section{Data validation}

Data on the Intellectual Disability Register have been comprehensively cleaned for people born 1983 onwards. ${ }^{4}$ Manual checks of clinical files were undertaken for older records if level of intellectual disability or Heber diagnosis was missing. Currently, $84 \%$ of cases of intellectual disability with a genetic basis are cytogenetically assessed: $12 \%$ of these have been assessed in more recent years only. Validation of the psychiatric case register was undertaken independently of this study. ${ }^{14}$

\section{Analysis of the data}

Several analyses used whole-of-population data. Others were restricted to two birth cohorts: individuals born 1950-64 and 1965-79. The use of two birth cohorts allowed for a more comprehensive assessment of the impact of period effects, such as changing diagnostic or service trends, on the data. Cohort years were selected to maximise the probability of cohort members passing through the most critical risk period for onset of psychosis (late adolescence to early adulthood), while minimising reliance on older data collections and classifications on the Intellectual Disability Register. At the time of data extraction (February 2003), the age range for the older birth cohort was 38-52 years and, for the younger birth cohort, 23-37 years. Calculation of incidence rate ratios and their confidence intervals was based on Rothman \& Greenland. ${ }^{17}$ All other analyses used SPSS 14.0 including chi-squared analyses, logistic regression to calculate odds ratios, and survival analysis to estimate confidence intervals for median age at first contact with services. Significance levels were set at $P<0.05$; Bonferroni correction was not applied given the exploratory and descriptive nature of the paper.

\section{Results}

The study database combined records from both registers and consisted of 245749 individuals: 232454 with a record of psychiatric illness only, 9074 with intellectual disability only, and 
4221 with co-occurring psychiatric illness and intellectual disability. Of the individuals with intellectual disability, $31.7 \%$ had a psychiatric disorder and $1.8 \%$ of individuals with a psychiatric illness had intellectual disability. Analysis by birth cohort produced figures similar to the full data-set, albeit increasing over time. Had we relied on the Intellectual Disability Register alone, only $12.3 \%$ of people with intellectual disability would have been identified as having psychiatric morbidity (1428 out of 11576 persons), and had we relied on the psychiatric case register alone, only $1.0 \%$ (2288 of the 235692 individuals with psychiatric illness, excluding 1281 people with intellectual disability alone) would have been identified as also having intellectual disability (Table 1).

To assess the impact of the inclusion of borderline intellectual disability, and to allow comparison with studies in which it is not included, the analysis was repeated without these cases. The percentage of individuals with a psychiatric illness among those with intellectually disability rose very marginally to $32.0 \%$ for the full database, and the percentage of those with an intellectual disability among individuals with a psychiatric illness fell slightly to $1.6 \%$.

The distribution of selected psychiatric disorders among people with intellectual disability, and the distribution of intellectual disability among people with selected psychiatric disorders are shown in Table 2.

\section{Dual diagnosis compared with intellectual disability alone}

Intellectual disability profiles of people with a dual diagnosis were compared with those for people with an intellectual disability and no co-occurring psychiatric illness. Level of handicap was differentially distributed depending on dual diagnosis status. Individuals with a dual diagnosis were significantly more likely to have IQ levels in the borderline and mild ranges $(64.3 \%$ of those with a dual diagnosis compared with $53.6 \%$ of those with intellectual disability alone) and less likely to be severely or profoundly affected (9.2\% compared with $18.3 \%$ ).

Individuals with a dual diagnosis had a different presumed aetiological basis to their intellectual disability compared with people with intellectual disability alone. In those with a dual diagnosis it was significantly less likely to be attributed to a genetic cause (odds ratio $(\mathrm{OR})=0.4,95 \%$ CI $0.3-0.5$ for both birth cohorts). In particular, Down syndrome was much less prevalent in those with dual diagnosis $(\mathrm{OR}=0.1,95 \%$ CI $0.1-0.2$ for both birth cohorts). For Down syndrome with co-occurring psychiatric illness, the most frequent disorder was 'psychiatric disturbance' to which no specific diagnosis was attached. Notably, in the wholeof-population data for Down syndrome there were only four individuals with a record of psychotic illness, including one with schizophrenia. On the other hand, pervasive developmental disorder (autism, Asperger or Rett syndrome, or unspecified) was significantly more frequent in the dual-diagnosis group than in the intellectually-disabled group ( $\mathrm{OR}=5.9,95 \% \mathrm{CI} 2.6-13.3$ for the 1950-64 birth cohort; OR=3.7, 95\% CI 2.1-6.7 for the 196579 birth cohort), and a sizeable proportion of this dual-diagnosis group $(43.3 \%$ of the $1950-64$ cohort and $21.6 \%$ of the $1965-79$ cohort) had had a lifetime-ever diagnosis of psychosis recorded. The aetiological attribution of intellectual disability is tabulated by dual diagnosis status in Table 3.

Most people with a dual diagnosis had made contact with disability services prior to contact with mental health services (82.8\% in the 1950-64 birth cohort and $62.7 \%$ in the $1965-79$ birth cohort). On average, it was another 10-11 years before they were seen by mental health services. At the same time, however, individuals with a dual diagnosis were making first contact with disability services later than those with intellectual disability alone: at a median age of 11 years compared with 7 years for the 1950-64 birth cohort, and at 7 years compared with 3 years for the 1965-79 birth cohort. The difference between the two cohorts may be largely artefactual as the Intellectual Disability Register was only started in 1953.

Finally, we examined mortality risk for people with a dual diagnosis compared with people with intellectual disability only. Mortality risk was significantly elevated in the intellectual disability only group in both birth cohorts, with mortality incidence rate ratios of 1.4 (95\% CI 1.1-1.8) for the 1950-64 birth cohort and 2.8 (95\% CI 2.1-3.6) for the 1965-79 birth cohort. The distribution of age at death also differed between groups with those with a dual diagnosis being significantly older at the time of their death than individuals with intellectual disability only. The mean difference was 13 years for the older cohort and 11 years for the younger cohort.

\section{Dual diagnosis compared with psychiatric illness alone}

When compared with people with psychiatric illness only, individuals with dual diagnosis were younger at the time of their first contact with psychiatric services and at their first (if any) in-patient admission (Table 4). They were more likely to have had an in-patient admission, and those who had been admitted to hospital had more admissions and had spent more days in hospital, all suggestive of a more severe psychiatric illness (Table 5).

The mortality risk for individuals with a dual diagnosis was significantly increased over that for people with psychiatric illness alone, with mortality rate ratios of 2.3 (95\% CI 1.9-2.8) for the 1950-64 birth cohort and 2.2 (95\% CI 1.7-2.9) for the 1965-79 birth cohort.

\section{Dual diagnosis and borderline intellectual disability}

Fourteen per cent of people with a dual diagnosis had an IQ level in the borderline range (70-74). This group was compared with the other dual diagnosis cases. The basis of intellectual disability in the borderline group was significantly less likely to be attributed to genetic or known biomedical origin including metabolic or teratogenic effects and birth defects $(12.1 \%$ v. $20.8 \%, P<0.001)$. This group was also significantly less likely to have associated epilepsy or to have pervasive developmental disorder or Down syndrome (there were no cases of Down syndrome). The distribution of schizophrenia and bipolar disorder was similar in both groups, but the borderline group was significantly more likely to include people with unipolar depression. Even though both groups had a similar rate of psychiatric admissions, the borderline group had spent only half as much time in hospital.

\section{Intellectual disability with co-occurring schizophrenia}

Over one in ten of individuals with dual diagnosis (485/4221) had intellectual disability with co-occurring schizophrenia (16.1\% of individuals with a dual diagnosis born $1950-64$ and $10.6 \%$ of those born 1965-79). The percentage of all individuals with intellectual disability who developed schizophrenia was $5.2 \%$ for the 1950-64 birth cohort and 4.5\% for the 1965-70 birth cohort. Conversely, $5.2 \%$ of all people with schizophrenia in the 1950-64 birth cohort and $3.7 \%$ in the 1965-79 birth cohort had intellectual disability (Table 2).

The disability profile for this dual-diagnosis group with schizophrenia contrasted sharply with that of individuals who had no co-occurring psychiatric illness, with three-quarters 

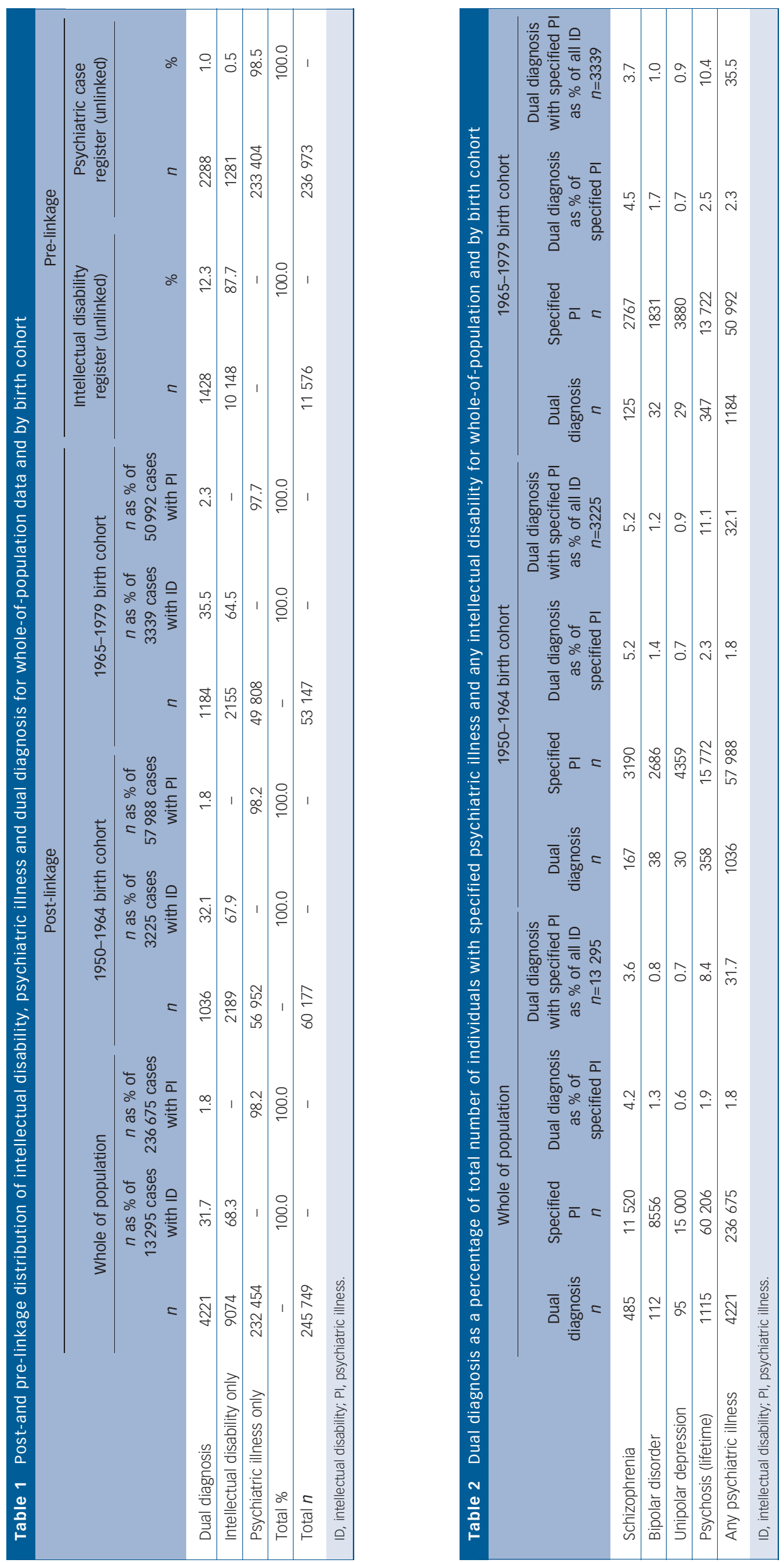
Table 3 Aetiological attributions of intellectual disability (ID) in individuals with a dual diagnosis compared with those with intellectual disability only, for whole-of-population and by birth cohort ${ }^{\mathrm{a}}$

\begin{tabular}{|c|c|c|c|c|c|c|c|c|c|c|c|c|}
\hline & \multicolumn{4}{|c|}{ Whole of population } & \multicolumn{4}{|c|}{ 1950-1964 birth cohort } & \multicolumn{4}{|c|}{ 1965-1979 birth cohort } \\
\hline & \multicolumn{2}{|c|}{ Dual diagnosis } & \multicolumn{2}{|c|}{ ID only } & \multicolumn{2}{|c|}{ Dual diagnosis } & \multicolumn{2}{|c|}{ ID only } & \multicolumn{2}{|c|}{ Dual diagnosis } & \multicolumn{2}{|c|}{ ID only } \\
\hline & $n$ & (\%) & $n$ & (\%) & $n$ & (\%) & $n$ & (\%) & $n$ & (\%) & $n$ & (\%) \\
\hline \multicolumn{13}{|l|}{ Aetiological factors } \\
\hline Chromosomal: Down syndrome & 97 & 2.3 & 1285 & 14.2 & 18 & 1.7 & 270 & 12.3 & 23 & 1.9 & 327 & 15.2 \\
\hline Chromosomal: sex-chromosome linked & 86 & 2.0 & 182 & 2.0 & 15 & 1.4 & 38 & 1.7 & 37 & 3.1 & 65 & 3.0 \\
\hline Chromosomal: other specified autosomal & 35 & 0.8 & 138 & 1.5 & 5 & 0.5 & 8 & 0.4 & 6 & 0.5 & 32 & 1.5 \\
\hline Genetic: other or not specified & 155 & 3.7 & 520 & 5.7 & 22 & 2.1 & 93 & 4.2 & 53 & 4.5 & 164 & 7.6 \\
\hline Metabolic disorders & 48 & 1.1 & 149 & 1.6 & 16 & 1.5 & 42 & 1.9 & 18 & 1.5 & 45 & 2.1 \\
\hline Teratogenic & 67 & 1.6 & 191 & 2.1 & 13 & 1.3 & 44 & 2.0 & 27 & 2.3 & 48 & 2.2 \\
\hline Central nervous system defect & 84 & 2.0 & 326 & 3.6 & 15 & 1.4 & 56 & 2.6 & 27 & 2.3 & 90 & 4.2 \\
\hline Other birth defect & 13 & 0.3 & 34 & 0.4 & 5 & 0.5 & 12 & 0.5 & 3 & 0.3 & 6 & 0.3 \\
\hline \multicolumn{13}{|l|}{ Other predisposing factors } \\
\hline Maternal medical condition & 79 & 1.9 & 167 & 1.8 & 20 & 1.9 & 58 & 2.6 & 26 & 2.2 & 50 & 2.3 \\
\hline Intra-uterine growth related & 81 & 1.9 & 219 & 2.4 & 16 & 1.5 & 32 & 1.5 & 29 & 2.4 & 68 & 3.2 \\
\hline Labour/delivery complications & 64 & 1.5 & 217 & 2.4 & 27 & 2.6 & 106 & 4.8 & 16 & 1.4 & 40 & 1.9 \\
\hline Perinatal complications & 157 & 3.7 & 435 & 4.8 & 51 & 4.9 & 119 & 5.4 & 43 & 3.6 & 102 & 4.7 \\
\hline Neonatal/postnatal complications & 252 & 6.0 & 565 & 6.2 & 76 & 7.3 & 173 & 7.9 & 70 & 5.9 & 168 & 7.8 \\
\hline Other including psychosocial factors & 391 & 9.3 & 648 & 7.1 & 109 & 10.5 & 164 & 7.5 & 103 & 8.7 & 168 & 7.8 \\
\hline No specified cause & 980 & 23.2 & 2505 & 27.6 & 266 & 25.7 & 750 & 34.3 & 236 & 19.9 & 511 & 23.7 \\
\hline \multicolumn{13}{|l|}{ Associated conditions } \\
\hline Epilepsy or convulsions & 664 & 22.0 & 1476 & 17.3 & 158 & 21.6 & 367 & 17.7 & 175 & 20.7 & 383 & 18.5 \\
\hline Cerebral palsy & 47 & 1.1 & 138 & 1.5 & 13 & 1.3 & 17 & 0.8 & 18 & 1.5 & 49 & 2.3 \\
\hline Sensory defect & 405 & 9.6 & 1113 & 12.3 & 84 & 8.1 & 241 & 11.0 & 107 & 9.0 & 294 & 13.6 \\
\hline Autism & 152 & 3.6 & 322 & 3.5 & 4 & 0.4 & 2 & 0.1 & 12 & 1.0 & 4 & 0.2 \\
\hline Asperger syndrome & 11 & 0.3 & 6 & 0.1 & 0 & - & 0 & - & 1 & 0.1 & 1 & 0.0 \\
\hline Rett syndrome & 14 & 0.3 & 27 & 0.3 & 1 & 0.1 & 2 & 0.1 & 4 & 0.3 & 11 & 0.5 \\
\hline $\begin{array}{l}\text { Pervasive developmental disorder } \\
\text { (unspecified) }\end{array}$ & 137 & 3.2 & 120 & 1.3 & 21 & 2.0 & 6 & 0.3 & 22 & 1.9 & 1 & 0.0 \\
\hline
\end{tabular}

(72.4\%) assessed as borderline or mild (compared with half of people solely with intellectual disability) and only $4.9 \%$ severely or profoundly affected. The diagnostic profile for those with intellectual disability with co-occurring schizophrenia also differed from that for people with schizophrenia only. Over their lifetime of contact with psychiatric services, individuals with a dual diagnosis ultimately diagnosed with schizophrenia were more likely initially to have been given diagnoses of paranoid psychoses $(33.2 \%$ v. 25.6\%); personality disorders (22.5\% v. $12.7 \%)$; psychotic and non-psychotic organic disorders $(25.2 \%$ v. 9.4\%); acute reaction to stress or adjustment reaction $(26.8 \% \quad v$. $12.9 \%)$; specific delays in development (14.6\% v. $0.8 \%)$; disturbance of conduct $(13.2 \%$ v. $2.1 \%)$; neurotic disorders $(21.0 \%$ v. $15.9 \%)$; and depressive disorders (15.3\% v. $7.9 \%)$. Moreover, this group was significantly younger at first contact with mental health services and at first admission compared with those with schizophrenia alone (Table 5). There was no difference between the two groups in mortality risk, with mortality rate ratios of 1.0 (95\% CI 0.6-1.7) for the 1950-64 birth cohort and 1.1 (95\% CI 0.5-2.5) for the 1965-79 birth cohort. However, individuals with a dual diagnosis were more likely to attempt suicide or serious self-harm $(24.0 \% v .17 .4 \%$ for the older birth cohort; $28.0 \%$ v. $19.6 \%$ for the younger birth cohort). For the most part, people with intellectual disability and co-occurring schizophrenia used psychiatric services more than the schizophrenia-only group, and more than the group with intellectual disability and co-occurring psychiatric illness of any kind. In the 1950-64 birth cohort, compared with individuals with schizophrenia only, cases of dual diagnosis with schizophrenia had a slightly higher admission rate but twice the cumulated total of in-patient days despite the fact that the percentage with at least one admission was similar for both groups. This pattern also held true for the 1965-79 birth cohort (Table 5).

\section{Discussion}

This work represents one of the most comprehensive whole-ofpopulation studies of the epidemiology of dual diagnosis. Using record linkage across health jurisdictions in Western Australia, we found that almost a third of individuals with intellectual disability had concurrent psychiatric morbidity, and nearly $2 \%$ of people with a psychiatric illness also had intellectual disability. Had the study relied on single register data, the figures would have been much lower, seriously underestimating the size of the problem of dual diagnosis in the population. Total psychiatric morbidity in intellectually disabled populations is comparable with general population estimates of lifetime prevalence of $41.2 \%{ }^{18}$ A recent paper reported a point prevalence of $40.9 \%$ for an intellectually disabled population, and estimated a likely range of $30-50 \% .^{7}$ Our figure may still be an underestimate due to the omission of cases seen by general practitioners as well as diagnostic overshadowing. There are few epidemiological studies of dual diagnosis for comparison in Australia or elsewhere. The only population data on psychiatric illness in adults who are intellectually disabled in Australia are 6-month prevalence estimates, with figures of $1.3 \%$ for psychotic disorders, $8 \%$ for depressive disorders and $14 \%$ for anxiety disorder. ${ }^{3}$ However, the use of lay interviewers employing an instrument not validated 


\begin{tabular}{|c|c|c|c|c|}
\hline & \multicolumn{2}{|c|}{ Age at first psychiatric contact } & \multicolumn{2}{|c|}{ Age at first in-patient admission } \\
\hline & $\begin{array}{l}\text { Dual diagnosis } \\
\text { Median }(95 \% \mathrm{Cl})\end{array}$ & $\begin{array}{l}\text { Psychiatric illness only } \\
\text { Median }(95 \% \mathrm{Cl})\end{array}$ & $\begin{array}{l}\text { Dual diagnosis } \\
\text { Median }(95 \% \mathrm{Cl})\end{array}$ & $\begin{array}{l}\text { Psychiatric illness only } \\
\text { Median }(95 \% \mathrm{Cl})\end{array}$ \\
\hline Schizophrenia & $23.8(22.6-24.9)$ & $30.1(29.7-30.5)$ & $24.5(23.1-25.9)$ & $30.4(30.0-30.8)$ \\
\hline Bipolar disorder & $26.9(25.1-28.7)$ & $35.0(34.6-35.4)$ & $30.0(27.4-32.6)$ & $35.6(35.1-36.1)$ \\
\hline Unipolar depression & $26.6(25.1-28.1)$ & $36.4(36.0-36.8)$ & $29.5(23.9-35.1)$ & $38.6(38.1-39.1)$ \\
\hline Psychosis (lifetime) & $23.6(22.8-24.4)$ & $35.5(35.3-35.7)$ & $24.8(23.9-25.6)$ & $36.7(36.4-36.9)$ \\
\hline Any psychiatric illness & $18.8(18.3-19.2)$ & $32.2(32.1-32.3)$ & $22.5(21.9-23.1)$ & $36.8(36.7-37.0)$ \\
\hline
\end{tabular}

for use with intellectually disabled samples (Composite International Diagnostic Interview) may have affected estimates. On the other hand, fourth-wave data for a cohort of Australian children who were intellectually disabled aged 19-31 indicated that $31 \%$ (down from $41 \% 11$ years earlier) met criteria for major psychopathology including behavioural and emotional problems using an instrument developed specifically for intellectually disabled populations. ${ }^{19}$

\section{Intellectual disability co-occurring with psychotic illness}

We found that, at $5.1 \%$ for the older cohort and $3.7 \%$ for the younger cohort, the prevalence of schizophrenia among individuals with an intellectual disability was not only at least three times higher than population lifetime estimates (most recently $1.26 \%),{ }^{20}$ but also higher than the commonly quoted estimate of $3 \%$ for schizophrenia among intellectually disabled populations. The prevalence of schizophrenia among people with intellectual disability was higher than that of bipolar disorder ( $1.2 \%$ for the older cohort, $1.0 \%$ for the younger cohort) and unipolar major depression $(0.9 \%$ for both birth cohorts) in the same population. The percentage of individuals with intellectual disability who had bipolar disorder was within general population estimates of 1.3 for Bipolar I and $\mathrm{II}^{21}$ while the percentage with unipolar depression was well below general population estimates (for example, $15.4 \%$ in the NEMESIS study). ${ }^{18}$ The estimate for a lifetime history of at least one episode of non-organic psychotic disorder among people who are intellectually disabled was one in ten $(11.1 \%$ of the older birth cohort and $10.4 \%$ of the younger birth cohort). This is markedly higher than general population estimates: recent epidemiological data from Finland put the lifetime prevalence of DSM-IV psychotic disorders at $2.43 \% .{ }^{20} \mathrm{~A}$ recent point prevalence estimate for intellectually disabled populations of $4.4 \%{ }^{7}$ adds credence to our high figure. Of note, the total figure for psychosis is largely attributable to people diagnosed with schizophrenia.

Compared with individuals with intellectual disability alone, those with dual diagnosis were more likely to have a diagnosis of pervasive developmental disorder, and far less likely to have Down syndrome. The latter finding was unexpected. Although the link between Down syndrome and Alzheimer's disease is well established, there is some evidence that psychosis may be rare in people with Down syndrome. ${ }^{22,23}$ Recent data suggest that the effect of 'unknown protective factors' associated with Down syndrome may extend to mental illness in general. ${ }^{24}$ Given how few studies have investigated this relationship directly, the question remains open as to whether the observed difference in prevalence is real or due to diagnostic under-ascertainment.

\section{The nature of the link}

The prevalence of intellectual disability among persons with schizophrenia, at $5.2 \%$ for the 1950-64 birth cohort and $4.5 \%$ for the 1965-79 birth cohort, was considerably higher than the Western Australian population estimate for intellectual disability of about $1.4 \%$. It is close to Kraepelin's original estimate ${ }^{25}$ that the basis of some $3.5 \%$ of cases of dementia praecox is mental retardation resulting in an early onset form of psychosis, which he called 'Pfropfschizophrenie' (engrafted schizophrenia). The high frequency of co-occurring schizophrenia and intellectual disability in our study poses a question as to the underlying basis of the association. Theories proposed include: a chance association; a de novo 'hybrid' condition; a severe schizophrenia; or a common aetiology. ${ }^{26}$ Since Kraepelin's early observations of cognitive impairment as an essential feature of dementia praecox cumulating evidence from clinical and epidemiological studies has provided compelling support for the latter view. Compromised intellectual ability in adolescence has been shown to be an independent risk factor for schizophrenia in prospective, population-based studies. ${ }^{27}$ Cognitive deficit involving several domains (verbal memory and learning, spatial working memory, attention, speed of information processing, performance IQ and motor skills), has been identified as a widely shared characteristic of schizophrenia in a comprehensive quantitative review. ${ }^{28}$ In a significant proportion of individuals, such deficits are present prior to onset of psychotic symptoms ${ }^{29}$ and remain stable over the course of illness, independent of clinical state. ${ }^{30}$ A similar, though attenuated, profile of deficit has been found in clinically unaffected first-degree relatives of people with schizophrenia and in unaffected co-twins from twin pairs discordant for schizophrenia. ${ }^{31}$ Such deficits tend to be accompanied by other early abnormalities affecting neuromotor function and language, ${ }^{32}$ suggesting a neurodevelopmental origin, long pre-dating psychosis. A re-analysis of Penrose's sample of families with mental illness and intellectual disability ${ }^{33}$ found a significant excess of relatives with diagnosis of schizophrenia among the probands with intellectual impairment. Together with findings that a specific genetic condition, 22q11 deletion syndrome, is associated with higher risks for both schizophrenia and learning disability, ${ }^{34}$ the evidence points to a likely common pathogenetic pathway for dual diagnosis. This is further supported by a comprehensive clinical and cognitive investigation of a sample of individuals with mild intellectual disability and schizophrenia. ${ }^{26}$ Recent research led by the senior co-author (A.J.) identified a distinct genetic basis for a subtype of schizophrenia characterised by pervasive cognitive deficit, comprising up to $50 \%$ of families with one or more affected members. ${ }^{35}$ The balance of evidence, therefore, favours a common aetiology for intellectual impairment and psychosis in a significant proportion of schizophrenia cases. 


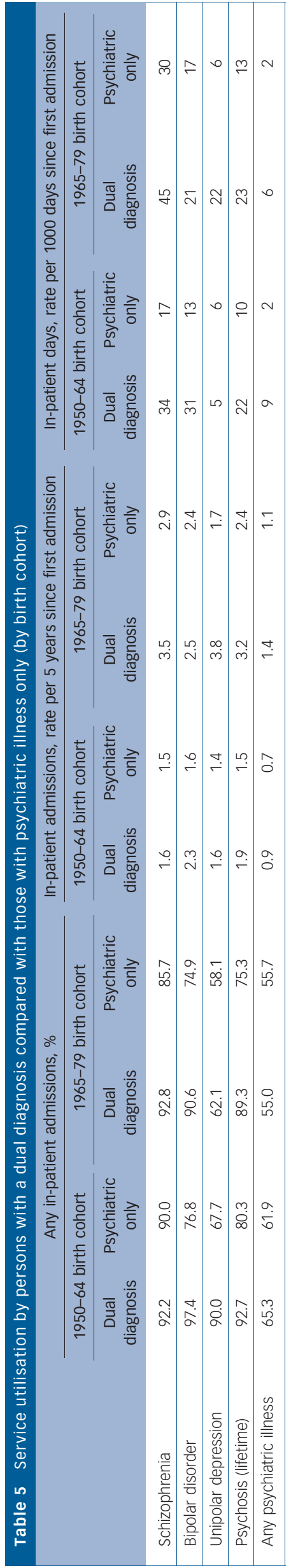

\section{IIIness severity and service use by people with co-occurring disorders}

In keeping with the literature, we found that people with a dual diagnosis had a more severe psychiatric illness than those with a psychiatric diagnosis alone ${ }^{36}$ including an earlier age at first contact with psychiatric services, more in-patient admissions and more in-patient days. These differences were particularly pronounced in those with schizophrenia co-occurring with intellectual disability. Indications that this is not an artefact arising from long periods of institutional care due to co-occurring intellectual disability include a higher median number of admissions in addition to a higher median number of in-patient days in the dual-diagnosis group. An alternative hypothesis, that the difference in severity is because only individuals with a dual diagnosis with the most severe psychiatric illness are able to access generic mental health services, could not be tested here.

\section{Limitations}

Although linkage of two registers resulted in an enriched source of data for analysis, there are several limitations related to the use of these administrative resources. First, it is likely that people with a dual diagnosis are still underestimated in these data. Several factors contribute to this underestimation. For one, the separation of mental health from intellectual disability services means that people with an intellectual disability may not be adequately assessed and treated for comorbid psychiatric illness; the converse also applies. Moreover, difficulties in clinical assessment may preclude the making of a psychiatric diagnosis in those with intellectual disability by masking psychiatric symptoms, especially less florid symptoms such as those associated with depression. Furthermore, the exclusion from the psychiatric register of general practice and private psychiatric contacts may lead to an underascertainment of individuals with intellectual disability who have co-occurring milder forms of mental illness. None the less, the inclusion of out-patient data makes the Western Australian psychiatric register one of the most comprehensive worldwide, and the psychoses, where lifetime contact with in- and out-patient services is high, will be particularly well represented. See, for example, prevalence estimates in the Australian National Survey of Low Prevalence (Psychotic) Disorders. ${ }^{37}$ Accordingly, this study focused primarily on the psychoses. Second, although the psychiatric register uses ICD criteria to record level of intellectual disability, no clear classification guidelines are in place for the recording of psychiatric comorbidity on the Intellectual Disability Register. Consequently, cases recorded as dual diagnosis on the Intellectual Disability Register were used only in the estimation of overall psychiatric comorbidity but could not be allocated to specific diagnostic categories. At most, these limitations may have led to an underestimate of the prevalence of dual diagnosis, without affecting the general pattern of findings and conclusions. The relative stability of many findings across the birth cohorts suggests that they are not artefacts of changing diagnostic trends and paradigms.

\section{The need for an integrated approach}

Both intellectual disability and severe psychiatric illness result in serious and lifelong impairments. Where the two co-occur, the impact of burden of disease on affected individuals, their carers, their family and friends, and the services that provide for them is particularly high. This study highlights the extent to which dual diagnosis is underestimated as a result of the administrative divide that has existed historically between services for people who are intellectually disabled and those for people with psychiatric illness. 
This is now increasingly acknowledged as having a profound effect on the capacity to care for individuals with dual diagnosis. ${ }^{38}$ In Western Australia, this division creates structural impediments to inter-agency approaches to integrated clinical practice: there is no specific service provision for people with a dual diagnosis, and mainstream psychiatric services are provided only upon referral. Of concern, correct identification of dual diagnosis is poor, measurement tools are still in nascence, ${ }^{39}$ and little research informs the treatment evidence base. ${ }^{40-41}$ This has impeded the development of interventions specifically for individuals with dual diagnosis and affected the ability of mental health professionals in both service arms to formulate a better basis for the routine management of these difficult cases, including early intervention programmes. In the absence of an integrated approach, people with dual diagnosis remain in an administrative gap, severely disabled, yet out of reach of best evidence-based practice.

Vera A. Morgan, MSocSc, Neuropsychiatric Epidemiology Research Unit, School of Psychiatry and Clinical Neurosciences, The University of Western Australia; Helen Leonard, MBChB, Jenny Bourke, MPH, Telethon Institute for Child Health Research, Centre for Child Health Research, The University of Western Australia; Assen Jablensky, DMSC, Neuropsychiatric Epidemiology Research Unit and Centre for Clinical Research in Neuropsychiatry, School of Psychiatry and Clinical Neurosciences, The University of Western Australia, Perth, Australia

Correspondence: Vera A. Morgan, The University of Western Australia Schoo of Psychiatry and Clinical Neurosciences, Level 3 Medical Research Foundation Building, Rear 50 Murray Street, Perth, Western Australia, Australia 6000. Email:vmorgan@cyllene.uwa.edu.au

First received 29 Aug 2007, final revision 3 Apr 2008, accepted 29 Apr 2008

\section{Acknowledgements}

We acknowledge Tom Pinder and Alan Joyce from the Western Australian Department of Health for implementing the diagnostic algorithm written by the first author (V.M.) and the Western Australian Data Linkage Unit for undertaking the linkage. Support for this research was provided by a University of Western Australia Research Grant to the first author (V.M.) and a donation by the Friends and Family of Andrew Catchpole.

\section{References}

1 Leonard $\mathrm{H}$, Wen $\mathrm{X}$. The epidemiology of mental retardation: challenges and opportunities in the new millennium. Mental Retard Dev Disabil Res Rev 2002; 8: 117-34.

2 Harris J. Intellectual Disability. Understanding its Development, Causes, Classification, Evaluation, and Treatment. Oxford University Press, 2006.

3 White P, Chant D, Edwards N, Townsend C, Waghorn G. Prevalence of intellectual disability and comorbid mental illness in an Australian community sample. Aust N Z J Psychiatry 2005; 39: 395-400.

4 Leonard H, Petterson B, Bower C, Sanders R. Prevalence of intellectual disability in Western Australia. Paediatr Perinat Epidemiol 2003; 17: 58-67.

5 Turner T. Schizophrenia and mental handicap: an historical review, with implications for further research. Psychol Med 1989; 19: 301-14.

6 Borthwick-Duffy SA. Epidemiology and prevalence of psychopathology in people with mental retardation. J Consult Clin Psychol 1994; 62: 17-27.

7 Cooper S-A, Smiley E, Morrison J, Williamson A, Allan L. Mental ill-health in adults with intellectual disabilities: prevalence and associated factors. Br J Psychiatry 2007; 190: 27-35.

8 Cooper S-A, Bailey NM. Psychiatric disorders among adults with learning disabilities: prevalence and relationship to ability level. Irish J Psychol Med 2001; 18: 45-53.

9 Penrose LS. A Clinical and Genetic Study of 1280 Cases of Mental Defect. Medical Research Council: Special Report Number 229. HMSO, 1938

10 Hemmings CP. Schizophrenia spectrum disorders in people with intellectual disabilities. Curr Opin Psychiatry 2006; 19: 470-4.

11 American Assocation on Mental Retardation. Mental Retardation: Definition Classification and Systems of Support (9th edn). American Association on Mental Retardation, 1992.

12 Heber R. A manual on terminology and classification in mental retardation. Am J Ment Defic 1959; 64: 1-111.
13 Yeargin-Allsopp M, Murphy C, Cordero J, Decouflé P, Hollowell J. Reported biomedical causes and associated medical conditions for mental retardation among 10-year-old children, metropolitan Atlanta, 1985 to 1987. Dev Med Child Neurol 1997; 39: 142-9.

14 Jablensky A, Morgan V, Zubrick S, Bower C, Yellachich L. Pregnancy, delivery, and neonatal complications in a population cohort of women with schizophrenia and major affective disorders. Am J Psychiatry 2005; 162 79-91.

15 World Health Organization. Mental Disorders. Glossary and Guide to their Classification in accordance with the Ninth Revision of the International Classification of Diseases. WHO, 1978

16 Kelman CW, Bass AJ, Holman CDJ. Research use of linked health data - a best practice protocol. Aust N Z J Public Health 2002; 26: 251-5.

17 Rothman KJ, Greenland S. Modern Epidemiology (2nd edn). Lippincott-Raven, 1998.

18 Bijl RV, Ravelli A, van Zessen G. Prevalence of psychiatric disorder in the general population: results of the Netherlands Mental Health Survey and Incidence Study (NEMESIS). Soc Psychiatry Psychiatr Epidemiol 1998; 33 587-95

19 Einfeld S, Piccinin A, Mackinnon A, Hofer S, Taffe J, Gray KM, Bontemp DE, Hoffman LR, Parmenter T, Tonge BJ. Psychopathology in young people with intellectual disability. JAMA 2006; 296: 1981-9.

20 Perälä J, Suvisaari J, Saarni S, Kuoppasalmi K, Isometsä E, Pirkola S, Partonen T, Tuulio-Henriksson A, Hintikka J, Kieseppa T, Härkänen T, Koskinen S, Löngvist J. Lifetime Prevalence of Psychotic and Bipolar I Disorders in a General Population. Arch Gen Psychiatry 2007; 64: 19-28.

21 Judd LL, Akiskal HS. The prevalence and disability of bipolar spectrum disorders in the US population: re-analysis of the ECA database taking into account subthreshold cases. J Affect Disord 2003; 73: 123-31.

22 Sovner R, Hurley A, Labrie R. Is mania incompatible with Down's syndrome? Br J Psychiatry 1985; 146: 319-20.

23 Collacott RA, Cooper SA, McGrother C. Differential rates of psychiatric disorders in adults with Down's syndrome compared with other mentally handicapped adults. Br J Psychiatry 1992; 161: 671-4.

24 Mantry D, Cooper SA, Smiley E, Morrison J, Allan L, Williamson A, Finlayson J, Jackson $A$. The prevalence and incidence of mental ill-health in adults with Down syndrome. J Intellect Disabil Res 2008; 52: 141-55.

25 Kraepelin E. Dementia Praecox and Paraphrenia. R. E. Krieger Publishers, 1919.

26 Doody GA, Johnstone EC, Sanderson TL, Owens DG, Muir WJ. 'Pfropfschizophrenie' revisited. Schizophrenia in people with mild learning disability. Br J Psychiatry 1998; 173: 145-53.

27 Reichenberg A, Weiser M, Rapp MA, Rabinowitz J, Caspi A, Schmeidler J, Knobler HY, Lubin G, Nahon D, Harvey PD, Davidson M. Elaboration on premorbid intellectual performance in schizophrenia: premorbid intellectual decline and risk for schizophrenia. Arch Gen Psychiatry 2005; 62: 1297-304.

28 Heinrichs RW, Zakzanis KK. Neurocognitive deficit in schizophrenia: a quantitative review of the evidence. Neuropsychology 1998; 12: 426-45.

29 van Winkel R, Myin-Germeys I, Delespaul P, Peuskens J, De Hert M, van Os J. Premorbid IQ as a predictor for the course of IQ in first onset patients with schizophrenia: a 10-year follow-up study. Schizophr Res 2006; 88: 47-54.

30 Caspi A, Reichenberg A, Weiser M, Rabinowitz J, Kaplan Ze, Knobler $\mathrm{H}$, Davidson-Saqi N, Davidson M. Cognitive performance in schizophrenia patients assessed before and following the first psychotic episode. Schizophr Res 2003; 65: 87-94.

31 Cannon TD, Huttunen MO, Lonnqvist J, Tuulio-Henriksson A, Pirkola T, Glahn D, Finkelstein J, Hietanen M, Kaprio J, Koskenvuo M. The inheritance of neuropsychological dysfunction in twins discordant for schizophrenia. Am J Hum Genet 2000; 67: 369-82.

32 Cannon M, Caspi A, Moffitt TE, Harrington H, Taylor A, Murray RM, Poulton R. Evidence for early-childhood, pan-developmental impairment specific to schizophreniform disorder: results from a longitudinal birth cohort. Arch Gen Psychiatry 2002; 59: 449-56.

33 Greenwood C, Husted J, Bomba M, Hodgkinson K, Bassett A. Elevated rates of schizophrenia in a familial sample with mental illness and intellectual disability. J Intellect Disabil Res 2004; 48: 531-9.

34 Bassett AS, Chow EW. 22q11 deletion syndrome: a genetic subtype of schizophrenia. Biol Psychiatry 1999; 46: 882-91.

35 Hallmayer JF, Kalaydjieva L, Badcock J, Dragovic M, Howell S, Michie PT, Rock D, Vile D, Williams R, Corder EH, Hollingworth K, Jablensky A. Genetic Evidence for a distinct subtype of schizophrenia characterized by pervasive cognitive deficit. Am J Hum Genet 2005; 77: 468-76.

36 Bouras N, Martin G, Leese M, Vanstraelen M, Holt G, Thomas C, Hindler C, Boardman J. Schizophrenia-spectrum psychoses in people with and without intellectual disability. J Intellect Disabil Res 2004; 48: 548-55. 
37 Jablensky A, McGrath J, Herrman H, Castle D, Gureje O, Morgan V, Korten A Harvey C. Psychotic disorders in urban areas: An overview of the methods and findings of the Study on Low Prevalence Disorders, National Survey of Mental Health and Wellbeing 1996-1998. Aust N Z J Psychiatry 2000; 34: 221-36.

38 Catinari S, Vass A, Ermilov M, Heresco-Levy U. Pfropfschizophrenia in the age of deinstitutionalization: whose problem? Compr Psychiatry 2005; 46: 200-5.
39 Holland AJ, Koot HM. Mental health and intellectual disability: an international perspective. Conference report. J Intellect Disabil Res 1998; 42: $505-12$.

40 Chaplin R. General psychiatric services for adults with intellectual disability and mental illness. J Intellect Disabil Res 2004; 48: 1-10.

41 Deb S, Weston S. Psychiatric illness and mental retardation. Curr Opin Psychiatry 2000; 13: 497-505.

\section{Poem}

\section{Wanting to die}

\section{Anne Sexton}

Since you ask, most days I cannot remember.

I walk in my clothing, unmarked by that voyage.

Then the almost unnameable lust returns.

Even then I have nothing against life.

I know well the grass blades you mention, the furniture you have placed under the sun.

But suicides have a special language.

Like carpenters they want to know which tools.

They never ask why build.

Twice I have so simply declared myself,

have possessed the enemy, eaten the enemy,

have taken on his craft, his magic.

In this way, heavy and thoughtful,

warmer than oil or water,

I have rested, drooling at the mouth-hole.

I did not think of my body at needle point.

Even the cornea and the leftover urine were gone.

Suicides have already betrayed the body.

Still-born, they don't always die,

but dazzled, they can't forget a drug so sweet

that even children would look on and smile.

To thrust all that life under your tongue!-

that, all by itself, becomes a passion.

Death's a sad bone; bruised, you'd say,

and yet she waits for me, year after year,

to so delicately undo an old wound,

to empty my breath from its bad prison.

Balanced there, suicides sometimes meet, raging at the fruit a pumped-up moon,

leaving the bread they mistook for a kiss,

leaving the page of the book carelessly open

something unsaid, the phone off the hook

and the love whatever it was, an infection.

From The Complete Poems of Anne Sexton (Houghton Mifflin, 1981). @1981 Linda Gray Sexton and Loring Conant, Jr. Reprinted with the permission of Sterling Lord Literistic, Inc.

Anne Sexton (1928-1974) was an American poet of the Confessional school. Throughout her life she had severe depression and was hospitalised on several occasions. She began writing poetry while recovering after a suicide attempt in 1956, as suggested by her therapist, Dr Martin Orne, and almost instantly won great acclaim - her first book, To Bedlam and Part Way Back (1960), was critically praised and nominated for a National Book Award. Sexton's poetry explored childhood guilt, mental illness, motherhood and female sexuality in a candid and unflinching way (she thought that poetry 'should almost hurt'), and is characterised by musical rhythms and striking imagery. She died by asphyxiating herself. 